\title{
Shape and Size Dependence of Electronic Properties of InSb Diamondoids and Nanocrystals: A Density Functional Theory Study
}

\author{
Mudar Ahmed Abdulsattar, ${ }^{1}$ Thamer R. Sultan, ${ }^{2,3}$ and Ahmed M. Saeed ${ }^{1}$ \\ ${ }^{1}$ Ministry of Science and Technology, Baghdad, Iraq \\ 2 The Higher Academy of Scientific \& Human Studies, Baghdad, Iraq \\ ${ }^{3}$ Ministry of Interior, Baghdad, Iraq
}

Correspondence should be addressed to Mudar Ahmed Abdulsattar; mudarahmed3@yahoo.com

Received 24 August 2013; Accepted 7 November 2013

Academic Editor: Bogdan Mihai Nae

Copyright (C) 2013 Mudar Ahmed Abdulsattar et al. This is an open access article distributed under the Creative Commons Attribution License, which permits unrestricted use, distribution, and reproduction in any medium, provided the original work is properly cited.

\begin{abstract}
Shape and size dependence of electronic properties of InSb diamondoids and nanocrystals is investigated using density functional theory. Cluster and large unit cell methods are combined with molecular orbital methods to obtain electronic structure of InSb diamondoids and nanocrystals. Starting from the simple molecules of hydrogenated InSb clusters such as $\operatorname{InSbH}_{6}, \mathrm{In}_{3} \mathrm{Sb}_{3} \mathrm{H}_{12}, \mathrm{InSb}_{-}$ diamantane, InSb-tetramantane, and InSb-hexamantane and ending with InSb large unit cell method we were able to obtain the electronic structure of a wide range of InSb nanostructures. Results showed that energy gap and In-Sb bond lengths generally decrease as the number of atoms increases with remarkable dependence on the shape of the molecule or nanocrystal. Atomic charges, tetrahedral angles, and bond lengths are used to compare different sizes, locations, and shapes of InSb diamondoids and nanocrystals.
\end{abstract}

\section{Introduction}

InSb is a unique semiconductor. It has one of the largest lattice constants and one of the smallest energy gaps. These properties give InSb the opportunity to cover some applications that no other semiconductor can be used for. As an example, the small energy gap $(0.17 \mathrm{eV}[1])$ nominated InSb to be used in infrared devices and other applications. These applications span photodiodes [2], thermal imaging [3], terahertz radiation [4], and so forth.

Transforming materials to their nanoscale size change, many of their properties. These changes include their electronic, mechanical, and optical properties. InSb nanoparticles undergo these changes that might include new applications as is the case for other materials. In the present work we introduce InSb diamondoids as a molecular limit and building blocks for larger nanocrystals. These molecules (diamondoids) are well known and found in nature as cage like molecules that resemble the tetrahedral bonding of carbon [5]. Cage like molecules other than carbon are found in nature or synthesized with a variety of atoms such as $\mathrm{Si}$, $\mathrm{P}$, and $\mathrm{N}$ compounds. We shall show that even numbered diamondoids (diamantane, tetramantane, and hexamantane) can also form stable molecules for the III-V InSb semiconductor compound. Odd numbered diamondoids (adamantane, triamantane, etc.) produce molecules with unequal number of In and Sb atoms.

\section{Theory}

Geometrical optimization method is used in the present work to obtain the electronic structure of InSb molecules and nanocrystals (Figure 1.) These include the following: $\mathrm{InSbH}_{6}$, $\mathrm{In}_{3} \mathrm{Sb}_{3} \mathrm{H}_{12}$, InSb-diamantane, InSb-tetramantane, and InSbhexamantane.

The large unit cell (LUC) method is a supercell method that can be used to model several sizes (Figure 2) of nanocrystals [6-8]. The LUC method differs from cluster method in 


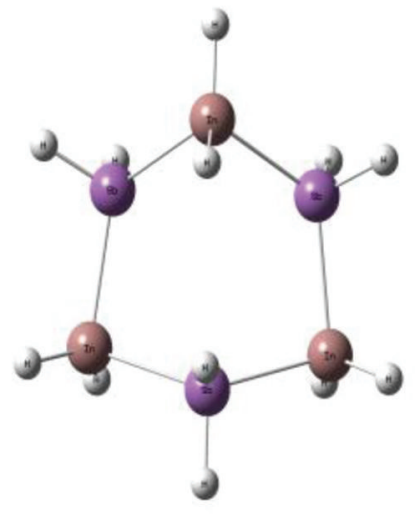

(a)

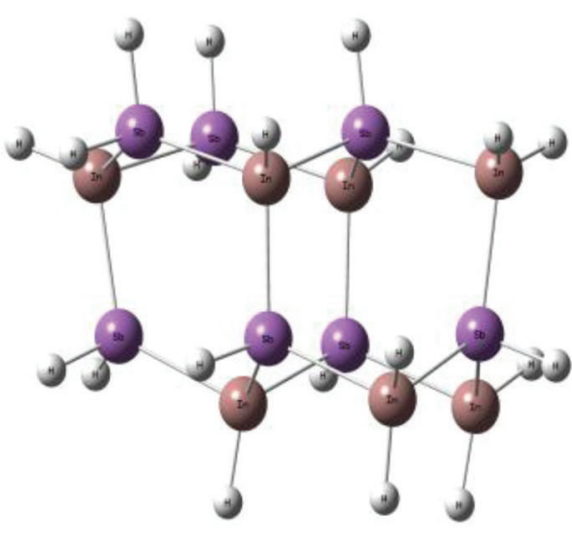

(b)

FIGURE 1: Shape of geometrically optimized (a) $\mathrm{In}_{3} \mathrm{Sb}_{3} \mathrm{H}_{12}$ and (b) InSb-diamantane. PBE/3-21G method is used for the present figure.

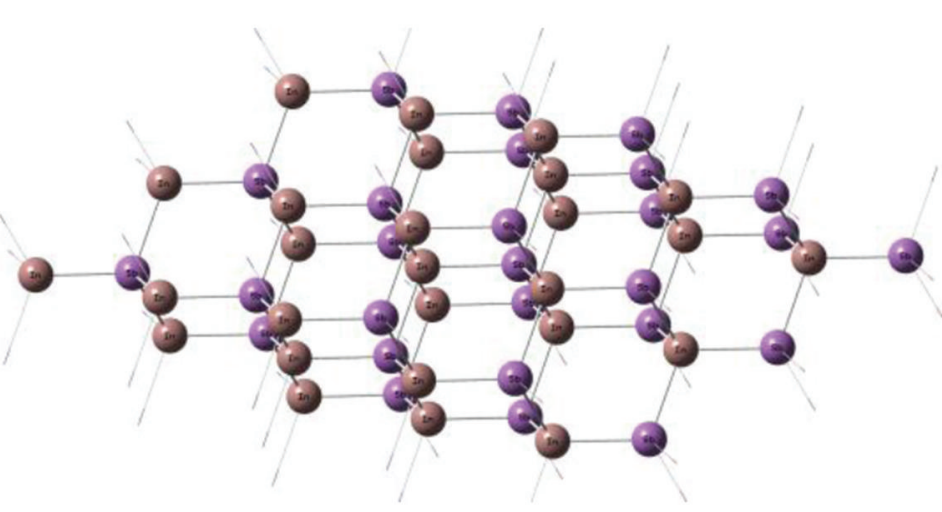

(a)

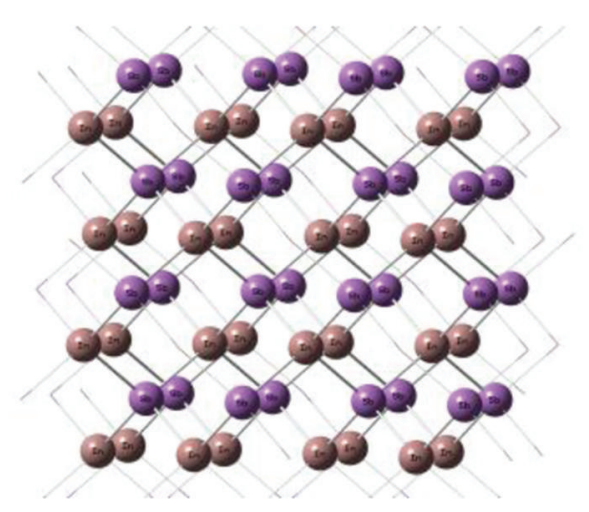

(b)

FIGURE 2: (a) 54-atom $\left(\mathrm{In}_{27} \mathrm{Sb}_{27}\right)$ and (b) 64-atom $\left(\mathrm{In}_{32} \mathrm{Sb}_{32}\right)$ LUC that represent the core of 470 and 512 combined In and Sb atoms cluster (after adding the periodically repeated cells), respectively. PBE/STO-3G method is used for the LUC calculations.

that it resembles the core part of the nanocrystal and neglects the surface part. It is different from other supercell methods by the fact that it uses $\mathbf{k}=0$ approximation ( $\mathbf{k}$ is the wave vector) with four-neighbor interaction range to resemble the core part of a nanocrystal. The four-neighbor range is chosen since surface reconstruction usually does not penetrate more than this distance [9]. Density functional theory (DFT) is used in the present work at the generalized gradient approximation level of Perdew, Burke and Ernzerhof (PBE). STO-3G and 3-21G bases sets are used as the basis functions of DFT calculations. More accurate basis such as $6-31 \mathrm{G}$ are unavailable for In or Sb elements in Gaussian 03 program which is used in the present work [10]. Since In and Sb are both heavy elements $(z \approx 50)$ relativistic effects must be included. Relativistic effects are included by adding spin-orbit corrections near the $\Gamma$ high symmetry points [11]. The present suggested InSb-diamondoids and LUC method are applied for the first time to InSb nanostructures. These methods can be compared with previous methods used for InSb such as pseudopotential or $\mathbf{k} \cdot \mathbf{p}$ methods $[12,13]$. Unlike the previous methods, the present two methods start from molecular sizes building up structures to reach the nanoscale region (bottomup methods) whereas the previous methods are essentially solid state methods reapplied to nanoscale sizes (top-down methods).

\section{Results and Discussion}

Figure 3 shows DFT calculated energy gaps using both cluster (cluster-DFT) and large unit cell (LUC-DFT) methods. These energy gaps are compared with bulk value $0.17 \mathrm{eV}$. In this figure we can note the dropping of the energy gap from nearly $4.5 \mathrm{eV}$ in InSb molecules $\left(\mathrm{InSbH}_{6}\right)$ using PBE/3-21G theory until it nearly stabilizes at nearly $2.49 \mathrm{eV}$ using PBE/STO$3 \mathrm{G}$ at high number of atoms using LUC-DFT method. An average gap reduction of $0.4 \mathrm{eV}$ between $3-21 \mathrm{G}$ basis and STO-3G basis can be seen in Figure 3. The oscillating behavior is due to shape effect of the different molecules used in present work. As an example, the two isomers InSb-tetramantane [121] and InSb-tetramantane [123] (both have the formula $\mathrm{In}_{11} \mathrm{Sb}_{11} \mathrm{H}_{28}$ ) have the two gaps 1.59 and $2.39 \mathrm{eV}$, respectively, using PBE/3-21G theory (see [14] for the [121] and [123] 


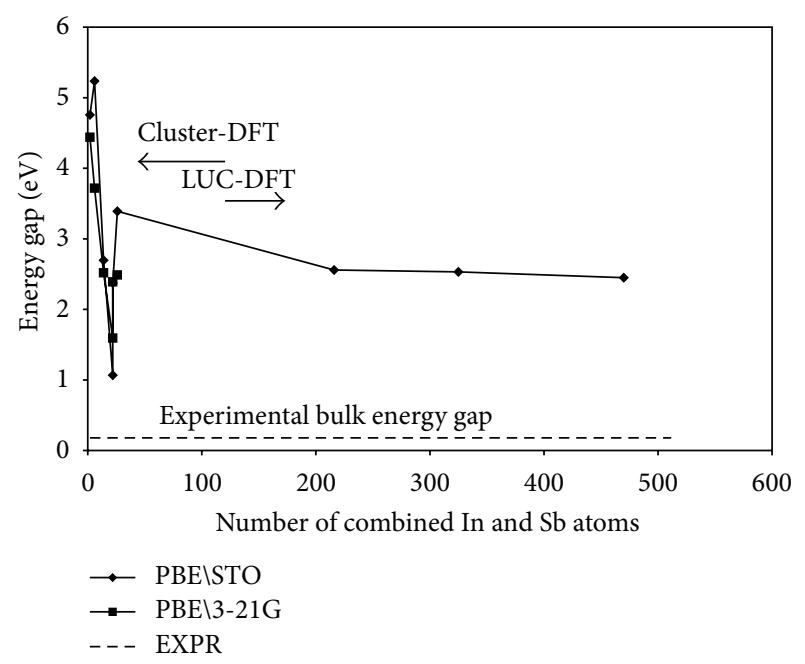

FIGURE 3: Energy gap as a function of combined number of In and $\mathrm{Sb}$ atoms. Relativistic corrections are incorporated in the present calculated values of the gap.

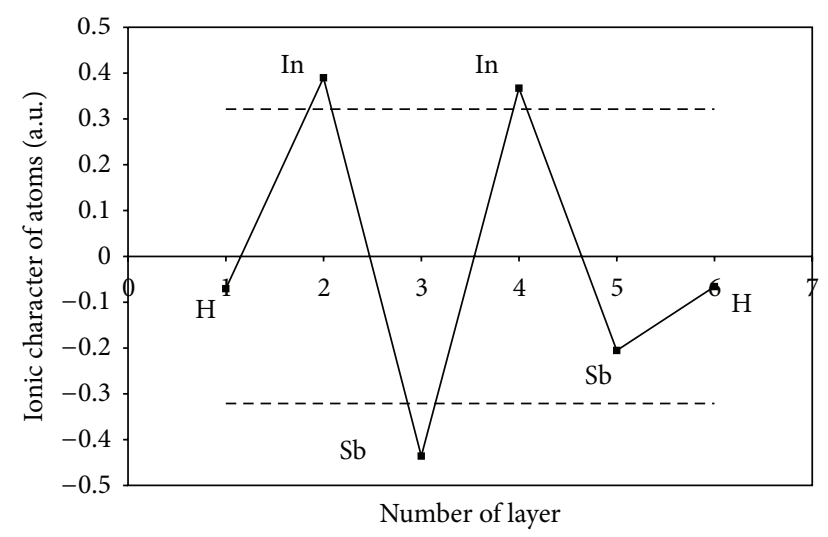

FIGURE 4: Atomic charges as a function of number of layers for InSbhexamantane. PBE/3-21G is used for the calculations of this figure. Experimental values of bulk InSb charges are shown as broken lines [9].

shape nomenclature of tetramantane). We included both 3$21 \mathrm{G}$ basis and STO-3G basis for the cluster-DFT results so that we can estimate the error in LUC-DFT theory which can be performed using STO-3G basis sets only. Using the average difference of $0.4 \mathrm{eV}$ mentioned above between 3-21G basis and STO-3G basis we expect that the highest nanostructure calculated in the present work $(2.5 \mathrm{~nm})$ has an energy gap of $2.09 \mathrm{eV}$ using 3-21G basis. Comparing this value with the experimentally obtained energy gap of $1.03 \mathrm{eV}$ for the $3.3 \mathrm{~nm}$ nanocrystals [15] we conclude that the present results are in the right direction of decreasing gaps as required by quantum confinement theory [16]. Unfortunately experimental results of InSb nanocrystals are rare but all these results confirm the present trend of values of decreasing gaps such as the $0.71 \mathrm{eV}$ gap of $6.5 \mathrm{~nm}$ nanocrystals [15].

Figure 4 illustrates atomic charges (ionic character) at every atom in a given path that connects two opposite sides

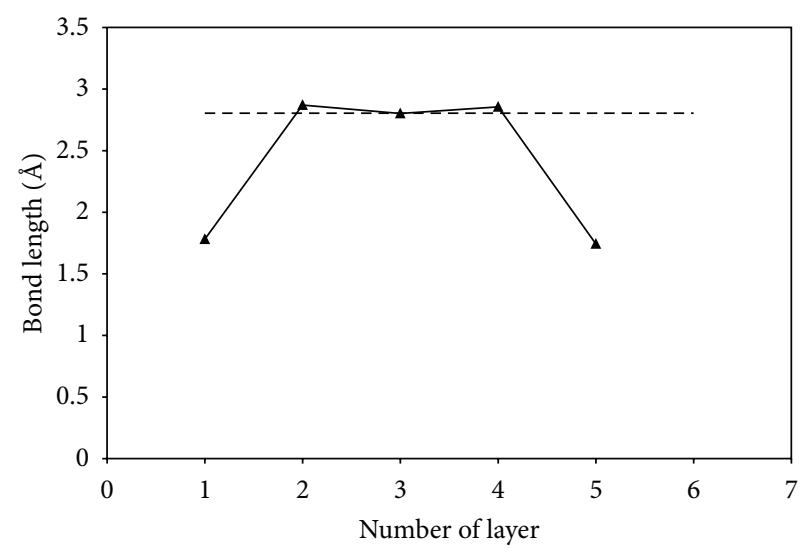

FIGURE 5: Bond lengths of InSb-hexamantane. PBE/3-21G theory is used for the calculations of this figure. The dashed line represents the experimental bulk value of In-Sb bond length [9].

of the InSb-hexamantane molecule. These charges are compared with experimental bulk ionic character charges of InSb [9] that have the value 0.32 atomic units (a.u.). In bulk InSb, In and Sb have the atomic charges 0.32 and -0.32 a.u., respectively, at core positions that are far from surface effects. This is changed at the surface due to symmetry breaking, higher electron affinity of hydrogen atoms, or other surface covering elements. At the left side of Figure 4, the hydrogen atom enhances the positive charge of In atom while it reduces the negative charge of the $\mathrm{Sb}$ atom at the right side.

Figure 5 illustrates the bond lengths using the same path of Figure 4. The two equal In-Sb bonds that are near the surface are mediated by a smaller In-Sb bond. $\mathrm{H}-\mathrm{In}$ and $\mathrm{H}-$ $\mathrm{Sb}$ bonds are nearly constant through all the molecules and are equal to 1.77 and $1.74 \AA$, respectively. This is expected since these bonds are localized only at the surface.

Figure 6 shows tetrahedral angles as a function of number of layers for InSb-hexamantane using the same path of Figures $4-5$. The tetrahedral angles range from $107.4^{\circ}$ to $111.5^{\circ}$ which is very close to the ideal value of this angle at 109.47 [17]. The value of this angle decreases slightly starting from the indium terminated surface and ending at the antimony terminated surface. The difference in electron affinity between In and $\mathrm{Sb}$ is the driving force that changes tetrahedral angles between the two ends.

Figure 7 shows In-Sb bond lengths as a function of number of atoms. After a high value is greater than $3 \AA$ at small molecules, this value decreases till it reaches $2.8 \AA$ for InSbdiamondoids. The present theory of LUC-DFT usually underestimates the value of bond lengths which is also the case for other LUC calculations [18]. The LUC-DFT limit is $2.68 \AA$ compared with the experimental value of $2.8 \AA$. Being less than $5 \%$ error is an expected trend in DFT calculations [19].

Figures 8 and 9 illustrate a sample of density of states of the various methods used in the present work. Figure 8 shows density of states of InSb-hexamantane while Figure 9 shows density of states of InSb 64 atoms LUC. From these figures one can determine the energy gap, width of valence and 


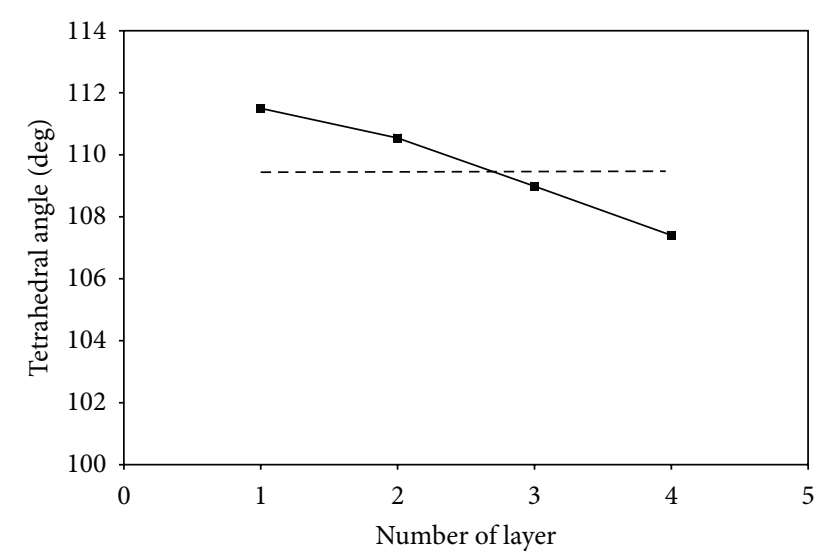

FIGURE 6: Tetrahedral angle as a function of number of layers for InSb-hexamantane. PBE/3-21G method is used for the calculations of this figure. The dashed line represents the ideal experimental bulk value of tetrahedral angle at 109.47 [18].

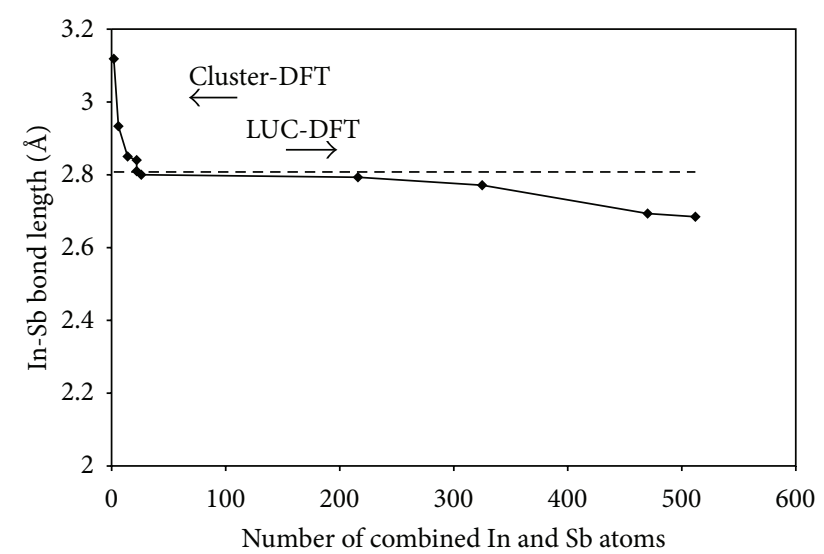

FIGURE 7: In-Sb bond length as a function of number of atoms for InSb molecules and nanocrystals. The dashed line represents the experimental bulk value of bond length [9].

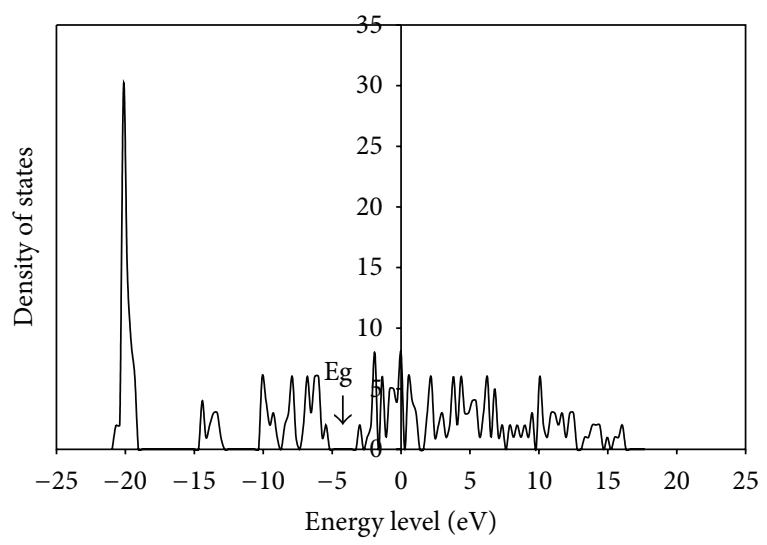

FIgURE 8: Density of states of InSb-hexamantane as a function of energy levels.

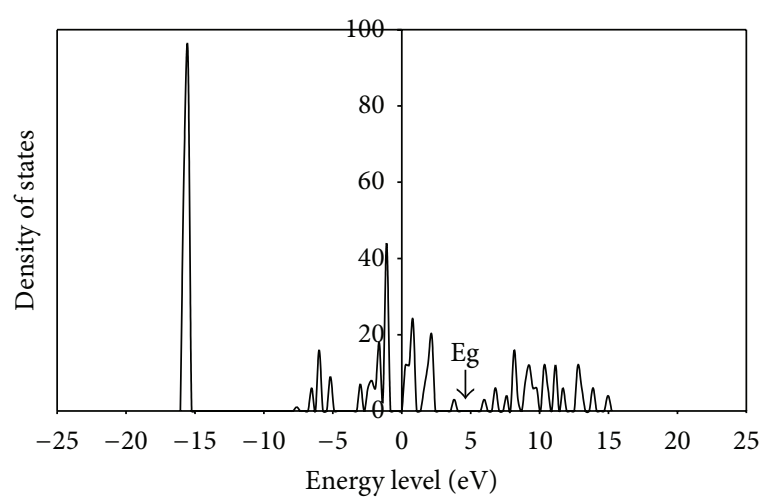

Figure 9: Density of states of InSb 64-atom LUC as a function of energy levels.

conduction bands, position of the highest occupied molecular orbital (HOMO), the lowest unoccupied molecular orbital (LUMO), Fermi level, gap intruder states, approximate ionization potential, and electron affinity. For comparison purposes we kept the energy range in these figures from -25 to $25 \mathrm{eV}$. The highest density of states in LUC is nearly three times of that of InSb-hexamantane molecule.

In addition to the sharper peaks in LUC method of Figure 9 with respect to Figure 8, one can note the movement of the whole energy spectra to more positive values. Remembering that LUC method represents the core part of the nanocrystal, one can conclude that hydrogen atoms at the InSb-hexamantane surface are the reason behind this movement. Hydrogen atoms with their higher electron affinity and tighter electron bonding drag the energy spectrum to deeper and stable energies with respect to the core part of InSb nanocrystal (represented by LUC method) that contains more free electrons. On the other hand, the higher symmetry of the core part is the reason behind the sharper and high quantum degenerate energy lines in LUC results which is usually reflected in sharper X-ray diffraction lines of nanocrystals.

\section{Conclusions}

InSb diamondoids are suggested in the present work as building blocks of InSb nanocrystals. Shape and size dependence of electronic properties of these blocks in comparison with LUC method results is investigated. Results show that shape and size of investigated structures play important role in their electronic properties. General reduction of energy gap and In-Sb bond lengths are seen with increasing particle size. This reduction is sometimes violated by shape effects. Density of states shows that electrons inside the core of $\mathrm{InSb}$ nanocrystals have more positive HOMO, LUMO, and Fermi levels with respect to diamondoid molecules. Density of states is sharper and higher at the center of the nanocrystal using LUC method. These states are less sharp and melt down at the surface due to hydrogen passivating atoms of diamondoids. In and $\mathrm{Sb}$ terminated surfaces differ in their bonds, charges, tetrahedral angles, and so forth. 


\section{Conflict of Interests}

All authors declare that they do not have any conflict of interests.

\section{References}

[1] P. D. Olszak, C. M. Cirloganu, S. Webster et al., "Spectral and temperature dependence of two-photon and free-carrier absorption in InSb," Physical Review B, vol. 82, no. 23, Article ID 235207, 2010.

[2] J. Abautret, J. P. Perez, A. Evirgen et al., "Electrical modeling of InSb PiN photodiode for avalanche operation," Journal of Applied Physics, vol. 113, Article ID 183716, 2013.

[3] K.-M. Chang, J.-J. Luo, C.-D. Chiang, and K.-C. Liu, "Wet etching characterization of InSb for thermal imaging applications," Japanese Journal of Applied Physics A, vol. 45, no. 3, pp. 14771482, 2006.

[4] V. L. Malevich, A. Krotkus, A. Biciunas, and V. Pacebutas, "Terahertz emission from femtosecond laser illuminated (112) surfaces of InSb," Journal of Applied Physics, vol. 104, Article ID 113117, 2008.

[5] L. Landt, K. Klunder, J. E. Dahl, R. M. K. Carlson, T. Moller, and C. Bostedt, "Optical response of diamond nanocrystals as a function of particle size, shape, and symmetry," Physical Review Letters, vol. 103, no. 4, Article ID 047402, 2009.

[6] H. M. Abduljalil, M. A. Abdulsattar, and S. R. Al-Mansoury, "SiGe nanocrystals core and surface electronic structure from ab initio large unit cell calculations," Micro and Nano Letters, vol. 6, no. 6, pp. 386-389, 2011.

[7] N. A. Nama, M. A. Abdulsattar, and A. M. Abdul-Lettif, "Surface and core electronic structure of oxidized silicon nanocrystals," Journal of Nanomaterials, vol. 2010, Article ID 952172, 6 pages, 2010.

[8] M. A. Abdulsattar, "Ab initio large unit cell calculations of the electronic structure of diamond nanocrystals," Solid State Sciences, vol. 13, no. 5, pp. 843-849, 2011.

[9] C. Kittel, Introduction to Solid State Physics, Wiley, 8th edition, 2005.

[10] Gaussian 03, Revision B.01, Gaussian Inc., Wallingford, Conn, USA, 2003.

[11] M. P. Surh, M. F. Li, and S. G. Louie, "Spin-orbit splitting of GaAs and InSb bands near $\Gamma$," Physical Review B, vol. 43, no. 5, pp. 4286-4294, 1991.

[12] C. Panse, D. Kriegner, and F. Bechstedt, "Polytypism of GaAs, InP, InAs, and InSb: an ab initio study," Physical Review B, vol. 84, no. 7, Article ID 075217, 2011.

[13] J. M. S. Orr, K.-C. Chuang, R. J. Nicholas, L. Buckle, M. T. Emeny, and P. D. Buckle, "Magnetoabsorption in InSb quantum-well heterostructures," Physical Review B, vol. 79, no. 23, Article ID 235302, 2009.

[14] J. Oomens, N. Polfer, O. Pirali et al., "Infrared spectroscopic investigation of higher diamondoids," Journal of Molecular Spectroscopy, vol. 238, no. 2, pp. 158-167, 2006.

[15] W. Y. Liu, A. Y. Chang, R. D. Schaller, and D. V. Talapin, "Colloidal InSb nanocrystals," Journal of the American Chemical Society, vol. 134, no. 50, pp. 20258-20261, 2012.

[16] M. A. Abdulsattar and K. H. Al-Bayati, "Corrections and parametrization of semiempirical large unit cell method for covalent semiconductors," Physical Review B, vol. 75, no. 24, Article ID 245201, 2007.
[17] H. N. Nasir, M. A. Abdulsattar, and H. M. Abduljalil, "Electronic structure of hydrogenated and surface-modified GaAs nanocrystals: Ab initio calculation," Advanced Condensed Matter Physics, vol. 2012, Article ID 348254, 5 pages, 2012.

[18] N. H. Aysa, M. A. Abdulsattar, and A. M. Abdul-Lettif, "Electronic structure of germanium nanocrystals core and (001)$(1 \times 1)$ oxidised surface," Micro and Nano Letters, vol. 6, no. 3, pp. 137-140, 2011.

[19] "NIST computational chemistry comparison and benchmark database," 2013, http://cccbdb.nist.gov/. 

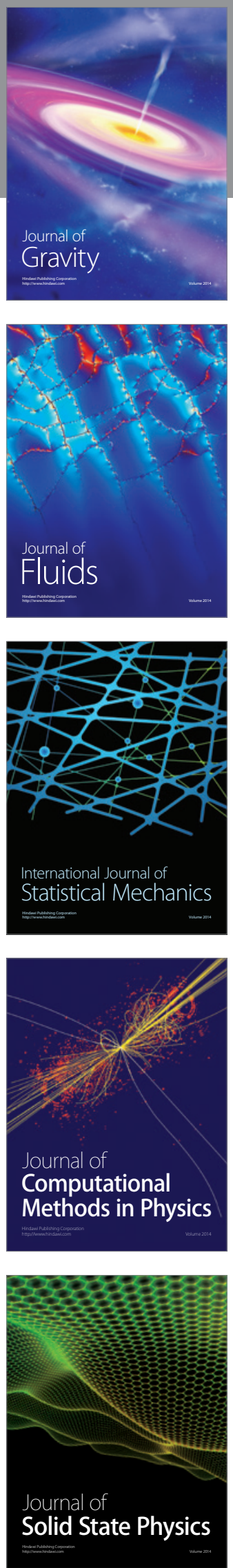

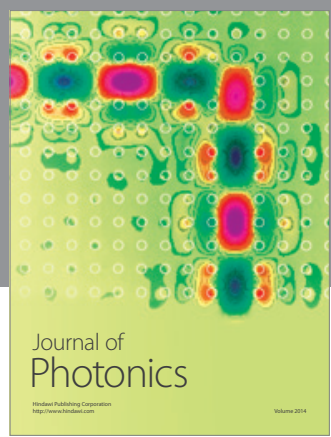

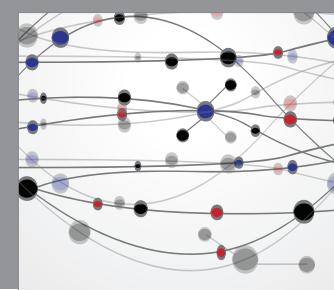

The Scientific World Journal

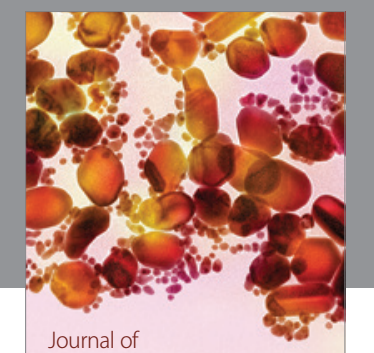

Soft Matter
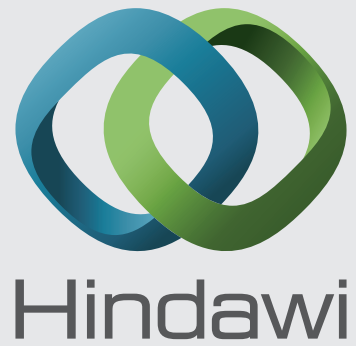

Submit your manuscripts at

http://www.hindawi.com
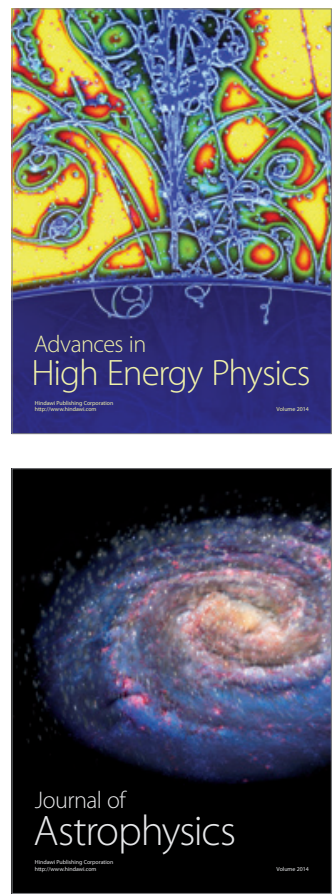
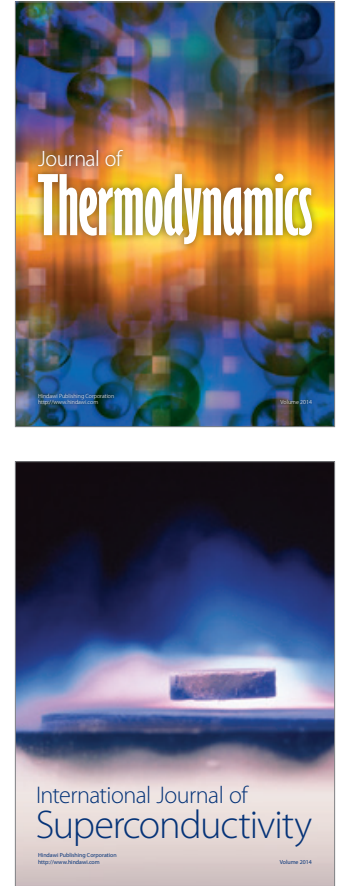
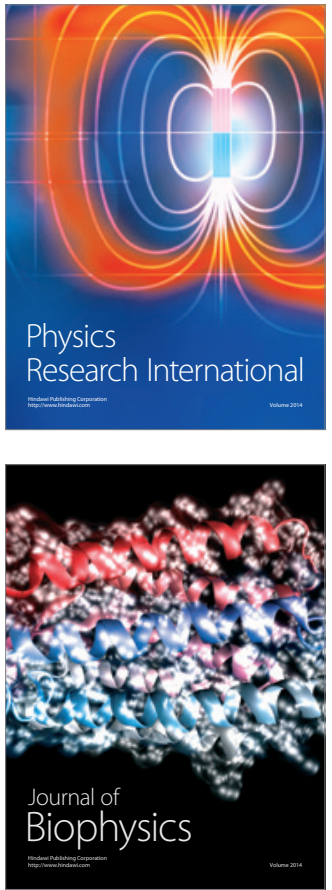
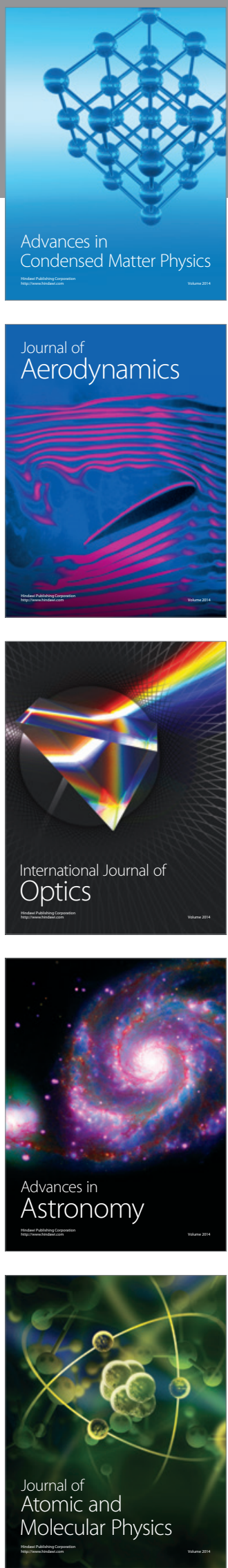